\title{
Linx
}

Revue des linguistes de l'université Paris X Nanterre

47 | 2002

Du sens au sens

\section{Sur le statut sémantique de il y a}

On the semantic status of Il y a

\section{Robert Martin}

\section{OpenEdition}

\section{Journals}

Édition électronique

URL : http://journals.openedition.org/linx/584

DOI : $10.4000 /$ linx.584

ISSN : 2118-9692

\section{Éditeur}

Presses universitaires de Paris Nanterre

\section{Édition imprimée}

Date de publication : 1 décembre 2002

Pagination : 79-87

ISSN : 0246-8743

\section{Référence électronique}

Robert Martin, «Sur le statut sémantique de il y a », Linx [En ligne], 47 | 2002, mis en ligne le 01 juin 2003, consulté le 30 avril 2019. URL : http://journals.openedition.org/linx/584 ; DOI : 10.4000/linx.584 


\title{
Sur le statut sémantique de $i l y a^{1}$
}

\author{
Robert Martin, Université de Paris-Sorbonne
}

Si la structure morpho-sémantique de il y a ne présente guère de difficulté ${ }^{2}$, il n'en est pas de même de son statut sémantique : $y$ avoir est-il un prédicat ou faut-il y voir un opérateur quantificationnel ? En classant il y a parmi les «présentatifs », les grammairiens laissent entendre que le verbe avoir n'a pas ici sa fonction ordinaire. Mais la question a agité surtout le débat philosophique et logique. Comme on sait, pour B. Russell, exister (et conséquemment $y$ avoir en français) ne saurait être un prédicat: exister (ou y avoir) entre dans la formulation du quantificateur logique dit « existentiel ».

Nous allons essayer de montrer que il y a, tout comme il existe, a en effet l'apparence d'un quantificateur. Mais, dans une approche linguistique, tout porte néanmoins à l'assimiler à un prédicat : même si l'existence est un prédicat à part de tous les autres, le traitement en termes de quantification paraît inapproprié pour de multiples raisons.

\section{Il y a (tout comme il existe) a l'apparence d'un quantificateur}

La forme « $\exists \mathrm{x}$ Px» se lit ainsi : «Il existe/il y a (au moins) un $\mathrm{x}$ tel que le prédicat $\mathrm{P}$ est vérifié pour $\mathrm{x} » \mathrm{ou}$, sous une forme plus courte : «il existe/il y a (au moins) un $\mathrm{x}$ tel que $\mathrm{Px} »$. La correspondance entre « $\exists$ » et il existe/il $y$ a est incontestable : il existe ou il y a fournissent de l'opérateur de quantification existentielle une parfaite traduction.

On observera tout de même d'entrée que la correspondance n'est pas exactement entre il existe ou ily a et « $\exists$ ». La traduction de « $\exists$ » est plutôt il existe un/il y a un et même il existe un... tel que/ily a un... tel que :

\footnotetext{
${ }^{1}$ C'est avec beaucoup d'émotion que je dédie ces quelques pages à la mémoire de Michel Galmiche : une grande sympathie de pensée nous unissait. Comment oublier la discrétion si attentive de Michel Galmiche, sa modestie profondément sincère, sa perspicacité toujours un peu inquiète et pourtant si constante ? Son sourire laissait deviner une immense bonté.

2 On se reportera à l'excellente description distributionnelle que procure M. Bichard, 1997. La plus grande difficulté est dans l'emploi quasi-prépositionnel : C'était il y a deux mois. Sur ce point, qui ne sera pas abordé ici, of. A. Henry, 1968, M. Wilmet, 1971 et N. Rivière 1993.
} 
Robert Martin

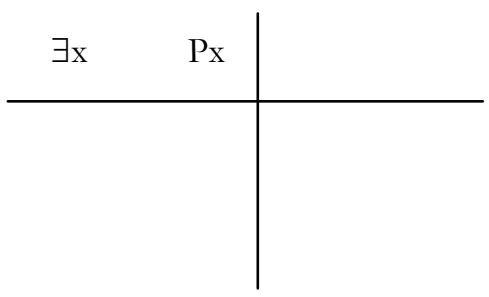

$$
\begin{aligned}
& \text { Il existe un } \mathrm{x} \quad \text { tel que } \mathrm{Px} \\
& \text { Ily a un } \mathrm{x} \quad \text { tel que } \mathrm{Px}
\end{aligned}
$$

C'est donc il existe un... tel que ou il y a un... tel que qui a l'apparence d'un quantificateur.

Comme on sait, le quantificateur existentiel de la logique des prédicats est en relation avec le quantificateur universel selon les équivalences suivantes :

$$
\begin{array}{cc}
\exists \mathrm{x} \text { Px } \Leftrightarrow & \sim \forall \mathrm{x} \sim \mathrm{Px} \\
\sim \exists \mathrm{x} \text { Px } \Leftrightarrow \quad \forall \mathrm{x} \sim \mathrm{Px} \\
\forall \mathrm{x} \text { Px } \Leftrightarrow \quad \sim \exists \mathrm{x} \sim \mathrm{Px} \\
\sim \forall \mathrm{x} \text { Px } \Leftrightarrow \quad \exists \mathrm{x} \sim \mathrm{Px} .
\end{array}
$$

Ce rapprochement de $« \exists »$ et de « $\forall$ » donne à entendre que le quantificateur fonctionne comme une extraction sur la classe des x. Il en est tout à fait ainsi dans les formes il existe un... tel que et ily a un... tel que.

- Dans le cas où l'existence est l'existence en soi, l'existence dans la réalité, dans le monde (allemand Sein), il y a est commutable avec il existe:

$$
\text { Il y a une maladie qui.../Il existe une maladie qui... ; }
$$

l'extraction opère sur la classe des maladies ; maladie est pris dans un sens générique et il y a une maladie telle que... réfère à une sorte de maladie.

Ailleurs, l'extraction opère, non pas sur une classe de variétés, de sortes, mais sur une classe d'individus :

$$
\text { Ily a une thèse sur.../Il existe une thèse sur... ; }
$$

ici thèse ne désigne pas un type, une sorte de thèses, mais un objet particulier et unique.

Que l'extraction soit générique, comme dans le premier cas, ou spécifique, comme dans le second, le mécanisme quantificationnel est le même, et c'est il y a un... (tel que) ou il existe un... (tel que) qui l'opèrent.

- Dans le cas où l'existence est non pas l'existence dans la réalité en général, mais l'existence occurrentielle, c'est-à-dire la simple présence ou la survenance dans une situation donnée (allemand Dasein), il y a un... tel que n'est pas commutable avec il existe un... tel que :

Ily a un moustique qui vient de me piquer/? Il existe un moustique qui vient de me piquer. 
Mais le mécanisme de la quantification existentielle demeure : le moustique en cause est extrait sur la classe des moustiques; on dit de lui deux choses : qu'il est là (il y a un moustique) et qu'il me pique (qui vient de me piquer).

Partout les apparences sont donc du côté de la quantification.

\section{Il y a marque des formes d'existence, des manières d'exister}

À y regarder de plus près, le dernier cas (il y a un moustique qui...) oriente cependant dans une direction bien différente. Il y a un $x$ tel que Px marque ici, outre la prédication $\mathrm{P}$ («tel que $\mathrm{P}$ »), le « être-là » du moustique. Or cet «être-là » peut fort bien occuper tout le champ de la prédication:

$$
\text { Ily a un moustique! }
$$

Cette phrase dit sans plus la présence de l'insecte indésirable. L'« occurrence», la «présence » est indifféremment celle d'un objet indéfini, non encore identifié, ou bien défini, déjà identifié :

$$
\begin{aligned}
& \text { Ily a un chat dans le jardin } \\
& \text { Ily a le chat dans le jardin. }
\end{aligned}
$$

Cette observation conduit tout naturellement à l'idée que dans il y a un... tel que, la quantification - c'est-à-dire l'extraction sur une classe - est opérée par un et que la fonction de ily a est autre : elle est de marquer l'« existence occurrentielle».

Dès lors, il nous faut revenir sur Il y a une maladie.../Il existe une maladie qui... et sur Il y a une thèse sur... / Il existe une thèse sur.... La fonction quantificationnelle n'est-elle pas, là aussi, assurée par le déterminant et non pas par ily a ou il existe ? Le rôle de ily a/ il existe n'est-il pas de signifier ici l' « existence en soi »?

Il n'est pas difficile de s'en convaincre. On se fondera là aussi sur des exemples où n'apparait pas, dans la même proposition, la forme «Px », c'est-à-dire où il n'y a pas de prédication sur l'objet extrait, en sorte que ily a (comme il existe) a pour seule fonction de marquer l'existence en soi. Il en est ainsi dans le type C'est comme cela depuis qu'il y a des hommes: l'extraction y est opérée par des sur la classe virtuelle de tous les hommes (ceux qui existent, qui ont existé, qui existeront, qui pourraient ou qui auraient pu exister) ; mais pas de « $\mathrm{Px}$ » ici; il y a a pour fonction unique de signifier l'existence en soi.

La même chose vaut pour Ily a un Dieu (et qui nous aime) ou bien S'ily a un Dien (il nous faut l'aimer plus que toute créature), mais avec une difficulté supplémentaire: sur quelle classe de référence opère l'article un ? On rapprochera ces phrases de Dieu existe ou de Si Dien existe. Exister, c'est être dans la réalité. Dien existe signifie que Dieu n'est pas un être conçu par la seule pensée, un « objet conceptuel », mais un être qui a une réalité en soi, indépendante de la pensée qui conçoit Dieu. Julien Sorel a existé signifie que Julien Sorel n'est pas un être de fiction, un être imaginé par la pensée, mais une personne qui a effectivement vécu. Bien entendu, la « réalité » ne peut être autre chose que ce que nous croyons être la réalité ; mais cette réalité est perçue comme distincte de ce que produit la seule pensée. Dire d'une chose qu'elle n'existe pas, c'est laisser entendre qu'elle n'a pas d'autre réalité que conceptuelle, qu'elle existe dans le discours, 
mais pas dans la réalité. Dire qu'elle existe, c'est affirmer au contraire qu'elle fait partie de la réalité, indépendamment de ce que nous en pensons ou des discours que nous en tenons.

Un commentaire en tout point comparable vaut pour Il y a un Dieu, et qui nous aime/Il existe un Dieu, et qui nous aime ou pour S'il y a un Dieu/S'il existe un Dieu ou encore S'il existe un paradis/S'il y a un paradis... Partout il s'agit d'évoquer l'existence dans la réalité. Sur quoi opère la quantification au moyen de un ? La classe de référence est ici celle des «objets conceptuels», des «êtres » que l'on désigne par dieu ou par paradis. La prédication exister ou y avoir dit qu'un objet extrait par un sur cette classe a une existence dans la réalité - une existence effective et non pas seulement conceptuelle ${ }^{3}$.

Tout porte donc à croire que la fonction de il y a est de marquer ou bien l'existence en soi ou bien l'existence occurrentielle, c'est-à-dire de signifier des manières d'exister, ce qui est de l'ordre de la prédication, et que, dans la forme il y a un... tel que (ou il existe un... tel que), la fonction proprement quantificationnelle est le fait du déterminant. En d'autres termes, il y a n'a que l'apparence d'un quantificateur, mais il n'est pas réellement un quantificateur.

\section{Il y a le $x$ a également un comportement de prédicat}

On comprend du même coup que ily a (et même il existe) soit compatible avec le défini ${ }^{4}$. $\mathrm{Il}$ a alors pour fonction d'activer discursivement un objet dont l'existence effective est présupposée. Cette existence est à nouveau l'existence en soi ou bien l'existence occurrentielle.

\subsection{L'existence en soi est rappelée}

- pour inviter à ne pas négliger ce qui existe, à ne pas l'oublier, à tirer les conséquences de ce qui est :

Il y a aussi la peur (" n'oublions pas que la peur existe; il est impossible de faire comme si elle n'existait pas »)

Et puis il y a la mort. Vous ne songez donc jamais à la mort, et que nous allons tous pourrir? J. Renard, Journal, 1910, p. 201) ${ }^{5}$;

- pour opposer la chose activée à une autre :

Il y a la bonne littérature et la mauvaise.

Ily a la fausse modestie, mais il n'y a pas de faux orgueil. (J. Renard, Journal, 1910, p. 1228)

${ }^{3}$ G. Kleiber, 1977 rejette l'interprétation quantificationnelle de exister (verbe intransitif), mais l'admet pour il existe. Le développement qu'on vient de lire va dans le sens du prédicat, même pour il existe.

${ }^{4}$ Nombreux exemples dans M. Bichard, 1997, en partic. p. 92 et suiv. et p. 110 et suiv.; 17 occurrences sont du type il y $a+$ tout... Le sens d'existence en soi peut même se trouver avec le quantificateur universel : Il y a bien / il existe bien toutes les misères que vous dites, mais ce n'est pas une raison pour désespérer.

${ }^{5}$ Les exemples référencés sont pris à la Base Discotext. 
Et dire qu'il y a la moitie des hommes comme toi... (Maupassant, Contes et nouvelles, t.1, p. 1061);

- pour répondre à une question qui invite à identifier des existants :

Quelles sont les planètes du système solaire? Il y a Neptune, Mars, la Terre...

Il s'agissait de déchainer l'enthousiasme. N'y a-t-il pas des procédés? Il y a la danse,

l'autosuggestion, les narcotiques, comme l'opium et le haschich, la boisson. (Barrès, Mes Cahiers, 1923, p. 13).

Dans tous ces emplois, il y a ne fait que rappeler l'existence d'un objet l'existence effective. Il n'est pas en mesure de la poser, à cause de la description définie qui la présuppose. Il y a Dieu ou Il y a la liberté ne peut pas signifier que Dieu existe ou que la liberté existe, mais seulement qu'il faut tenir compte de l'existence, présupposée, de Dieu ou de la liberté. La présupposition existentielle est dans la " description définie », la réactivation existentielle dans il y a.

On notera que il existe n'est pas exclu de tels emplois, à condition que l'article défini soit nettement générique. Il existe la bonne littérature et la mauvaise; Il existe beureusement en France la liberté de penser; Entre le joueur du matin et le joueur du soir, il existe la différence qui distingue le mari nonchalant de l'amant pâmé sous les fenêtres de la belle (Balzac, La Peau de chagrin, p. 5).

3.2. Dans le type Il y a le facteur, la fonction de il y a est double : il signifie une existence occurrentielle, et ce type se rapproche ainsi de Le facteur est là. Mais alors que dans Le facteur est là, l'objet identifiable Le facteur appartient à l'univers actuel du discours, dans Il y a le facteur, cet objet, également identifiable et qui existe, était précédemment hors du propos, c'est-à-dire transitoirement hors de la pensée active ; il y $a$ a à nouveau pour rôle de le réactiver.

3.3. Enfin, dans le type il y a que (Il y a que ce pauvre facteur a été victime d'un accident), il y a pose la réalité d'un contenu propositionnel.

Tout paraît donc plaider en faveur de l'hypothèse que il y a (tout comme il existe) a fondamentalement pour fonction de poser ou de réactiver des formes d'existence ${ }^{6}$ :

- l'existence dans la réalité (par opposition à l'existence purement conceptuelle)

- l'existence occurrentielle (c'est-à-dire la présence ou la survenance) ${ }^{7}$.

\footnotetext{
${ }^{6}$ L'idée d'existence s'affirme aussi dans le type temporel C'était il y a deux mois : il y a marque la réalité d'un intervalle temporel.

${ }^{7}$ La limite est floue entre l'existence en soi (l'existence dans la réalité) et l'existence occurrentielle (la simple présence). On glisse imperceptiblement du côté de l'existence occurrentielle, qui rend impossible il existe. Ainsi on dira indifféremment

Il y a une pharmacie / un cabinet médical / une étude notariale à Saint-Jean-les-choux

ou Il existe une pharmacie / un cabinet médical / une étude notariale à Saint-Jean-les-choux,
} 
Robert Martin

La quantification existentielle se situe du côté du déterminant et non pas de ily $a$, dont le rôle n'est autre que celui d'un prédicat.

\section{Il y a a linguistiquement toutes les propriétés du prédicat impersonnel}

On ne s'étonnera pas ainsi que $y$ avoir possède, linguistiquement, toutes les marques du prédicat.

4.1. Y avoir se conjugue à tous les temps et à tous les modes :

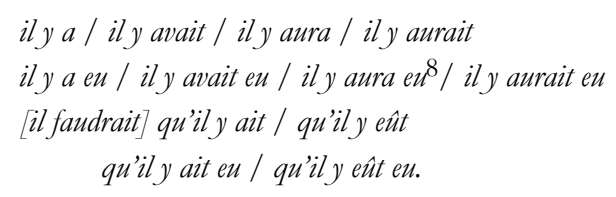

Le verbe étant linguistiquement le prédicat par excellence, y compris dans la tournure impersonnelle (qui nierait à pleut dans il pleut le statut de prédicat ?), les présomptions sont fortes pour que $y$ avoir soit un prédicat comme tous les verbes semblent l'être.

4.2. Y avoir est modalisable comme le sont tous les prédicats.

mais seulement Il y a un pharmacien / un médecin / un notaire à... (et non Il existe un pharmacien... à... ) ; l'existence en soi d'un pharmacien ne peut être circonscrite à un lieu ou à des circonstances particulières.

De même on dira Il y a ou bien Il existe une solution, une issue, une méthode, un procédé, un moyen, une façon de faire, une technique...

mais seulement: Ily a un avantage

$$
\text { Il y a de l'illusion à tout cela (et non *il existe). }
$$

La solution, l'issue, la méthode... ont une existence indépendante du lieu ou du moment, une existence perçue comme non contingente. L'avantage ou l'illusion, qui sont de l'ordre du jugement, de l'appréciation, ne sont pas au contraire ressentis comme existant en soi. Et dès lors, seul il y a est possible. Il existe ne devient acceptable (et encore... ) que dans un contexte de généricité : On peut se "pacser» à bon compte. Mais il existe tout de même, du côté du mariage, un avantage incontestable... .

La facilité et la diversité d'emploi de il y a conduit forcément à des ambiguités polysémiques auxquelles il existe échappe. Ainsi la question Existe-t-il un train pour Saint-Jean-les-choux ? équivaut à Saint-Jean-les-choux est-il desservi par un train? La même question avec $Y$ a-t-il a par ailleurs le sens de $L e$ train pour Saint-Jean-les-choux circule-t-il aujourd'bui / ce matin / ce soir... ?. Existence ou occurrence? Les deux sont envisageables avec il y $a$. Autre exemple : Il y a pire. Deux interprétations sont possibles :

- «On peut imaginer pire ; il peut exister pire » (existence en soi);

- «Je n’ai pas encore rapporté le pire de ce qui est arrivé » (existence occurrentielle).

${ }^{8}$ Voici, prises à Discotext, quelques attestations de cette forme rare :

Il y aura eu quelque accident. (Zola, La Conquête de Plassans, p. 1118),

J'aime le passé, mais je porte envie à l'avenir. Il y aura eu de l'avantage à passer sur cette planète le plus tard possible. Descartes serait transporté de joie s'il pouvait lire quelque chétif traité de physique et de cosmographie écrit de nos jours. (Renan, Souvenirs d'enfance et de jeunesse, p. 1),

Un tel état semblera un retour à l'âge primitif: mais entre les deux, il y aura eu des siècles d'étude patiente et attentive. (Renan, L'Avenir de la science, p. 309). 


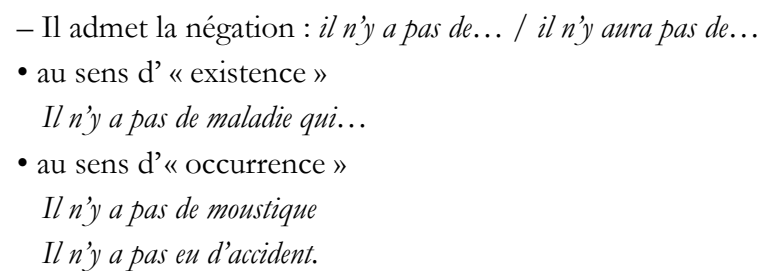

Une difficulté notable concerne le type Il y a le facteur qui arrive, c'est-à-dire la tournure où l'objet activé (le facteur) est en même temps prédiqué (qui arrive). La négation peut y affecter cette prédication :

Il y a le facteur qui n'arrive pas «Il y a ceci : le facteur n'arrive pas / n'arrive toujours pas », mais la négation de il y a ne s'opère qu'au prix d'une modification du déterminant :

Il n'y a pas de facteur qui arrive

ou bien, mais difficilement, par la négation de la phrase reprise en écho et immédiatement corrigée :

?Non, il n'y a pas le facteur qui passe, mais le médecin qui arrive.

(on dirait évidemment bien mieux: Non, ce n'est pas le facteur qui passe, mais...).

Ce type se rapproche de

Il y a que le facteur a été victime d'un accident,

où la négation de il y a que est carrément impossible. Cela se conçoit : il y a que ayant pour fonction de poser la réalité d'un contenu propositionnel, la négation aurait pour conséquence paradoxale de détruire cette fonction même.

- Y avoir admet l'interrogation et l'hypothèse :

- Est-ce qu'ily a une maladie qui...?

Est-ce qu'ily a un moustique?

Est-ce qu'ily a eu un accident?

- S'ily a une maladie qui...

S'ily a un moustique...

S'ily a un accident... .

Mais impossible de dire: *Est-ce qu'il y a que...? ou *S'il y a que... : ce serait à nouveau mettre en cause la fonction même qui est dévolue à il y a que.

4.3. Enfin, $y$ avoir fonctionne comme n'importe quel prédicat impersonnel. Dans le tour impersonnel, la "personne d'univers» (il $)^{9}$ sert d'ancrage à la prédication; celle-ci consiste avant toute chose à marquer une existence, en soi ou occurrentielle : Il broutait dans le pré un troupeau de vaches normandes, « il y avait dans le pré un troupeau de vaches normandes qui broutait». La congruence est donc parfaite

${ }^{9}$ Cf. G. Moignet, 1969. 
entre la prédication d'existence signifiée par il y a et la fonction dévolue à la tournure impersonnelle.

\section{Pas de correspondance bi-univoque entre « $\exists \mathrm{x} »$ et $I l y$ a un $x$}

On notera enfin qu'en tout état de cause il est impossible d'établir aucune correspondance bi-univoque entre « $\exists \mathrm{x}$ » et il $y$ a un $x$.

5.1. Il y a un $x$ est une paraphrase de « $\exists \mathrm{x}$ », mais parmi d'autres possibles. De même que « $\forall \mathrm{x}$ » se lit «pour tout $\mathrm{x} », 《 \exists \mathrm{x}$ » peut se lire «pour (au moins) un $\mathrm{x}$ ». Quand la négation porte sur le quantificateur $(\sim \forall \mathrm{x})$, on lit «pas pour tout $\mathrm{x}$ »; cette lecture a son pendant du côté de l'existentiel $(\sim \exists \mathrm{x})$ : certes * «pas pour (au moins) un $\mathrm{x}$ » n'est pas acceptable, mais ce contenu n'est autre que celui de «pour aucun $\mathrm{x}$ ». Soit

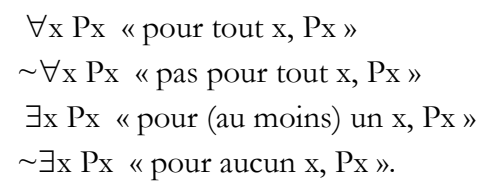

5.2. Inversement, il est impossible de considérer que il y a un $x$ représente, par excellence, la quantification existentielle du langage ordinaire. S'il en était ainsi, il y a un $x$ figurerait implicitement sous toute occurrence du déterminant existentiel. Il n'en est évidemment rien. Il voudrait épouser une Portugaise blonde qui sache parfaitement le japonais: qui dit que cette Portugaise existe? La classe des Portugaises n'est assurément pas vide, mais l'individu extrait sur cette classe virtuelle de Portugaises n'est d'aucune manière compatible avec ily a ou il existe, qui supposent l'existence dans la réalité. Il y a sous-jacent rendrait de tels emplois paradoxaux, puisque il $y$ a marque une existence effective (en soi ou occurrentielle). Si donc il y a un était un quantificateur, ce serait seulement un quantificateur parmi d'autres, certainement pas le quantificateur existentiel en soi. En fait, la fonction de ily a est ailleurs : c'est un prédicat existentiel.

Tout un faisceau d'arguments donne donc à penser que $y$ avoir a le statut d'un prédicat. Le prédicat d'existence est certes un prédicat particulier: présupposé par tous les autres, il est étroitement lié aux déterminants et aux mécanismes référentiels ; on ne s'étonne pas qu'il puisse apparaitre dans la paraphrase du quantificateur existentiel. Mais ce n'est qu'une paraphrase parmi d'autres, et linguistiquement, $y$ avoir, tout comme exister ou il existe, a toutes les caractéristiques du prédicat.

Robert MARTIN

Université de Paris-Sorbonne

1 , rue Victor-Cousin

75230 PARIS Cedex 05

eveline.martin@wanadoo.fr 
Sur le statut sémantique de $\mathrm{Il}$ y a

\section{BIBLIOGRAPHIE}

BICHARD M., 1997, Plaidoyer en faveur d'un mal-aimé : étude morpho-syntaxique de il y a en français contemporain, Lille, Septentrion, ("Thèses à la carte »).

GALMICHE M., 1991, Sémantique linguistique et logique (Un exemple: la théorie de R. Montague), Paris, PUF.

HENRY A., 1968, C'était il y a des lunes, Paris, Klincksieck.

KAWAGUCHI J., 1991, Avoir et les problèmes de la localisation en français, Tokyo, France Tosho.

KLEIBER G., 1977, « Sur le statut sémantico-logique du verbe exister », Trav. Ling., Littér. XV1, pp. 317-336.

MARTIN R., 1993, «Sur le paradoxe de la prédication d’inexistence », Fr. mod. t. 61, pp. 1-10.

MOIGNET G., 1969, « Le verbe Voici - Voilà », Trav. Ling. Littér., VII-1, pp. 189-202.

MOIGNET G., 1970, «Personne humaine et personne d'univers», Trav. Ling. Littér., VIII-1, pp. 190-202.

RIVIERE N., 1993, «Il y a : il y a 10 ans, il y a 10 ans que, depuis 10 ans », Modèles linguistiques t. 14, pp. 121-152.

RUSSELL B., 1970, Introduction à la philosophie mathématique, trad. fr.par G. Moreau, Paris, Payot.

SCHILLER A., 1992, Die Présentatifs im heutigen Französisch, Frankfurt am Main, P. Lang.

WAGNER R.-L., 1964, « Il y a », Le fr. dans le monde, 29, pp. 10-15.

WILMET M., 1971, "Note sur l'évolution sémantique et syntaxique de il y a », Trav. Ling. Littér., IX-1, pp. 283-308.

ZASLAWSKY D., 1974, «La philosophie analytique », in : Histoire de la philosophie, t. III, Paris, Gallimard, (La Pléiade), pp. 750-795 (en partic. pp. 771-782). 